\title{
Rectus Sheath Haematoma
}

\section{Lt Col S Chawla}

MJAFI 2002; 58 : 355-356

Key Words : Abdominal wall; Haematoma; Rectus sheath

\section{Introduction}

$\mathbf{R}$ ectus sheath haematoma is an accumulation of blood in the sheath of the anterior rectus abdominis muscle. This is caused by the rupture of epigastric vessels or by the tearing of the fibres of the rectus abdominis muscles [1]. It may follow minimal trauma such as coughing, bending or during sudden severe muscle contraction. Age group varies between 25-65 years, sex ratio female : male being 1:2.5. Accurate diagnosis should be made to prevent an unnecessary laparotomy since bleeding into rectus sheath can produce a clinical picture of an acute abdomen. 2 patients of rectus sheath haematoma who were diagnosed and managed successfully are presented here.

\section{Case Report-1}

24 year old serving soldier presented with severe pain in the lower abdomen following sudden jerk while getting down from a 3 ton vehicle. The pain was severe but without radiation, and it was exacerbated during coughing spells. There was no external bruise seen on abdominal wall. Firm tender lump was palpable in the hypogastric region on the right side. Laboratory tests showed haemoglobin as 13.5 gm\% and leukocyte count $12,300 / \mathrm{cmm}$. Ultrasound examination revealed small haematoma in the right rectus sheath (Fig-1). Conservative management was started with broad spectrum antibiotics and analgesics without much relief. Ultrasound guided needle aspiration of the haematoma was done and $10 \mathrm{ml}$ of dark coloured blood was aspirated. Patient recovered fully and on follow up there was no recurrence of haematoma.

\section{Case Report-2}

52 year old patient allegedly sustained injury due to fall when he was gored by a cow. He developed swelling of the hypogastric region. Initially it was considered to be full bladder but swelling persisted even after catheterization. Swelling gradually increased in size. Ultrasound examination revealed rectus sheath haematoma (Fig-2). Ultrasound guided needle aspiration was done and $50 \mathrm{ml}$ of serosanguinous fluid was drained. Patient developed recurrence of swelling. Subsequently surgical evacuation of haematoma was done and inferior epigastric vessel was ligated. Wound was closed in layers with tube drain which was removed after 24 hours. Patient showed uneventful postoperative recovery and was asymptomatic on follow up.

\section{Discussion}

Although uncommon, patients of rectus sheath haematoma are still seen in surgical practice. Usually it presents as an acute surgical problem and in some cases it may result in an unnecessary laparotomy [2]. It is a rare but important cause of abdominal pain. It occurs following trauma, infection, debilitating diseases, collagen diseases, pregnancy and puerperium. The predisposing factors include hypertension, atherosclerosis, old age, obesity, previous surgery, anticoagulant use and blood dyscrasias. Iatrogenic causes like laparoscopy and accidental intramuscular injection of subcutaneous low dose heparin in the lower abdominal wall have also been described by some authors [3].

Rectus sheath haematoma usually occurs below the umbilicus as was seen in these patients. Anatomically.

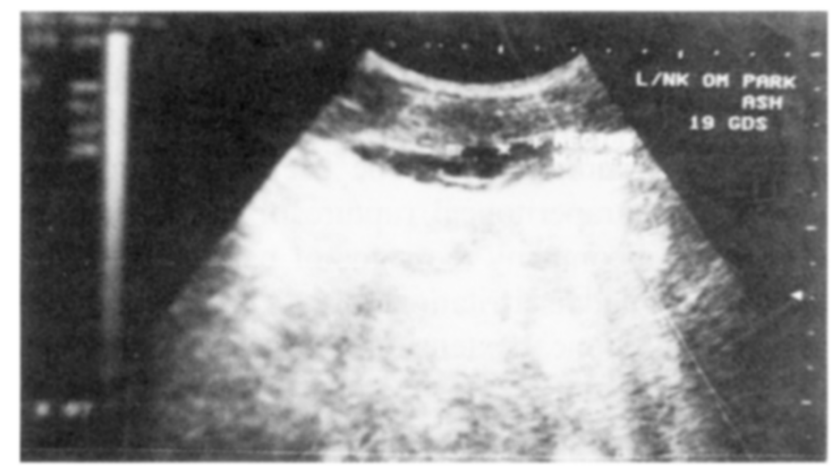

Fig. 1: Ultrasound abdomen showing small rectus sheath haematoma in a serving soldier

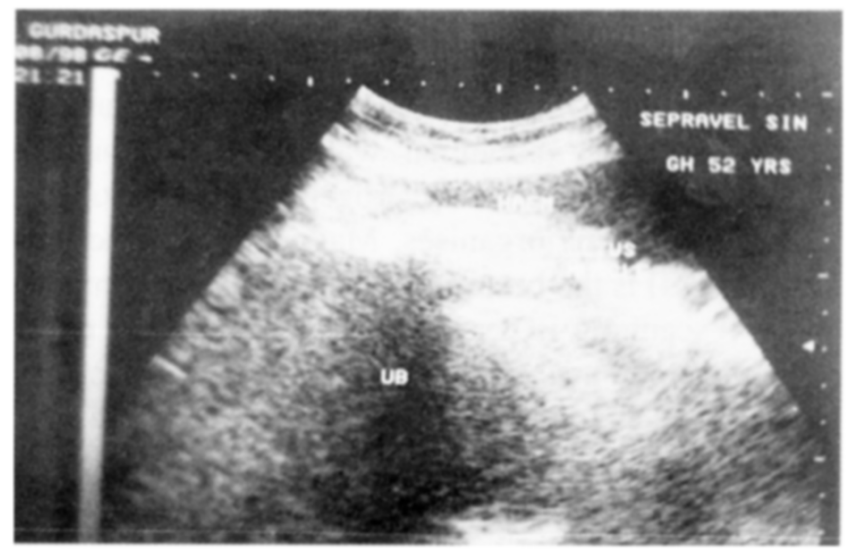

Fig. 2 : Ultrasound abdomen showing small rectus sheath haematoma in $\mathbf{5 2}$ year old patient 
in lower abdomen, below the linea semicircularis, only a weak transversalis fascia and peritoneurn support the rectus abdominis muscle. The inferior epigastric artery and vein pierce the rectus abdominis and are firmly attached to it. Muscle length changes during muscular contraction in flexion and extension, mainly in the lower half of the muscle leading to shearing forces at branch points of the inferior epigastric artery. Previous surgical scars also redirect these forces and predispose to haematoma formation [4]. Although cases have been reported where it may occur in the upper abdomen [5]. Diagnosis is usually clinical. Characteristically patient presents with sudden, sharp and severe pain followed by palpable mass in the upper or lower abdomen. In atypical cases, it may be confused with acute appendicitis and the palpable mass be mistaken for intra-abdominal abscess. Teske et al reported that out of 100 cases of rectus sheath haematoma, only $17 \%$ had been correctly diagnosed preoperatively [6]. In our patients, correct diagnosis was arrived at after initial evaluation of patients except in one case where surgical evacuation was required due to increasing size of haematoma despite aspiration. In another patient conservative management was successful. Suspicion of appendicitis may lead to unnecessary surgery in such patients. Fothergill's sign consists of raising the patients head end of the bed which either fixes an abdominal wall mass or makes an intra-abdominal mass less pronounced, can be useful. Cullen's sign implies an intraperitoneal rupture of the haematoma and would accompany the signs of parietal peritoneal irritation or bladder irritation. Grey Turner's sign suggests extraperitoneal extension. Both signs take four to six days to present.

Imaging is essential in determining the nature of the mass. Soft tissue lateral decubitus $X$-rays may have an early role but ultrasound and computerised tomographic (CT) scanning help in defining the lesion. Ultrasound in both patients was $100 \%$ sensitive. Ultrasound shows a fusiform longitudinal mass, usually confined to the abdominal wall, which is initially sonolucent but becomes heterogenous and sonodense as the haematoma organises. Magnetic resonance imaging (MRI) is the best modality for soft tissue masses and $C T$ scanning is very helpful in diagnosing rectus sheath haematomas; facilities for both these investigations were lacking. Ultrasound should be the investigation of choice as it is extremely sensitive, quicker and the more readily available imaging technique with no radiation exposure. It is also helpful in excluding uterine/ovarian pathology, monitoring the size of the haematoma by serial scanning and if percutaneous drainage is necessary as was done in one of the patients. Recent reports identify coughing, straining and sudden twisting to one side as the most common cause of this disease. In females it is common during pregnancy, but in our experience all patients were male, the common cause being blunt/indirect trauma.

In summary, diagnosis of rectus sheath haematoma is usually easy and treatment is simple, provided the possibility of this condition is kept in mind. At times in unusual presentations it often mimics many other intra-abdominal conditions and remains unrecognised until the patient is on the operation table. If the haematoma is small and no mass is palpable, ultrasound and CT scan are the procedures of choice for its correct diagnosis [7,8]. Treatment of rectus sheath haematomas has changed over the years and it is now generally accepted that if there is no haemodynamic compromise, the pain can be controlled, there are no peritoneal or urinary symptoms, and the haematoma is not enlarging then a conservative approach is acceptable as was followed in case 1. This would consist of bedrest, compression of the haematoma, icepacks and treatment of any predisposing causes. Surgical management consists of an open evacuation of the haematoma and ligation of any bleeding vessels as was done in case 2. Gelfoam embolization has also been reported as an effective and less invasive method of securing a bleeding inferior epigastric artery. Laparascopic approaches have also been described in cases of iatrogenic injury following laparoscopic port insertion. Awareness and knowledge of the condition and precipitating factors will allow the surgeon to effectively utilise noninvasive methods to confirm the diagnosis and manage this condition successfully in a peripheral setup.

\section{References}

I. Zainea CG, Jordan F. Rectus sheath haematoma. Pathogenesis, diagnosis and management. Am J Surg 1988;54:630-3.

2. Lohle PNM, Puylaert JBCM, Coerkamp EG et al. Nonpalpable rectus sheath haematoma clinically masquerading as appendicitis : US and CT diagnosis. Abdom Imaging 1995;20:152-4.

3. Casey RG, Mahmoud M, Carroll K et al. Rectus sheath haematoma : an unusual diagnosis. Irish Medical Journal 2000;93:90-2.

4. Yokota T, Yamada Y, Takahashi $M$ et al. Rectus sheath haematoma : what is your diagnosis? Indian J Surg 1999;61:326-8.

5. Bema JD, Gracia V. Guirao J et al. Rectus sheath haematoma : diagnostic classification by CT. Abdom lmaging $1996 ; 21: 62-4$.

6. Teske JM. Haematoma of the rectus muscle : case report and analysis of 100 cases from literature. Am J Surg 1946;71:689-95.

7. Ray CE, Wilbur AC. CT diagnosis of congnuent haematomas of psoas and rectus sheath: case report and review of anatomy, pathogenesis and imaging. Clinical Imaging 1993;17: 22-6.

8. Gallego AM, Aguayo JL. Flores B et al. Ultrasonography and computed tomography reduce unnecessary surgery in abdominal rectus sheath haematoma. Br J Surg 1997:84: I 295-7. 